\title{
FILTERED ALGEBRAS AND REPRESENTATIONS OF LIE ALGEBRAS ${ }^{1}$ )
}

\author{
BY \\ R. SRIDHARAN
}

Introduction. There is a general question as to how much can be said about a filtered object through the knowledge of its associated graded object. We consider here a particular case of this general problem. We take the symmetric algebra $S(L)$ of a free $K$-module $L$ and look for filtered $K$-algebras whose associated graded algebras are isomorphic to $S(L)$. Some such algebras are already known. In fact if $g$ denotes an arbitrary Lie algebra on $L$, the "Poincare-Witt Theorem" asserts that the universal enveloping algebra of $g$ is one such. It turns out that this gives "almost" a general solution of our problem. Indeed, the algebras we seek are suitable generalizations of the usual enveloping algebras and can in fact be defined as universal objects for certain "generalized representations" of Lie algebras on $L$. The rest of our results are on the cohomology of these algebras.

For a Lie algebra $g$ over a commutative ring $K$ and a 2-cocycle $f$ on its standard complex with values in $K$, we define in $\$ 1$ the notion of an $f$ representation. The usual representations of $\mathfrak{g}$ correspond to the case $f=0$.

We introduce in $\S 2$ the filtered $K$-algebra $g_{f}$ which is a universal model for $f$-representations. We deduce the "Poincaré-Witt Theorem" (Theorem 2.6) for $\mathfrak{g}_{f}$ as an easy consequence of the usual Poincaré-Witt Theorem, proved in [1, p. 271]. It is then clear that if $g$ is $K$-free, there is a graded $K$-algebra isomorphism $\psi_{f}: S(\mathfrak{g}) \rightarrow E^{0}\left(\mathfrak{g}_{f}\right)$, where $S(\mathfrak{g})$ denotes the symmetric algebra of the $K$-module $\mathfrak{g}$ and $E^{0}\left(g_{f}\right)$ the graded algebra associated with $g_{f}$ (Theorem 2.5).

In $\S 3$, we define, for a fixed graded $K$-algebra $S$, the category whose objects are pairs $\left(\Lambda, \psi_{\Lambda}\right)$, where $\Lambda$ is a filtered $K$-algebra, $\psi_{\Lambda}: S \rightarrow E^{0}(\Lambda)$ an isomorphism of graded algebras and whose maps are defined in an obvious manner. If $S$ is the symmetric algebra of a free $K$-module $L$, then there is a 1-1 correspondence between isomorphism classes of such objects and pairs $(\mathfrak{g}, f)$, where $\mathfrak{g}$ is a Lie algebra on $L$ and $\boldsymbol{f} \in H^{2}(\mathfrak{g}, K)$. For a cocycle $f$ in the class $\boldsymbol{f}$, the pair $\left(\mathfrak{g} f, \psi_{f}\right)$ is an object in the class corresponding to $(\mathfrak{g}, f)$ (Theorem 3.1).

The fourth section is devoted to the computations of certain of the usual homology and cohomology groups of a finite dimensional Lie algebra g. This amounts to a study of $\mathfrak{g} f$ for $f=0$. These computations are used in the next

Received by the editors January 5, 1961.

(1) This paper forms the substance of a doctoral thesis submitted to the Faculty of Pure Science, Columbia University. Its preparation was sponsored in part by the Office of Naval Research (NONR 266(57)). 
section to get the homology and cohomology of $\mathfrak{g}_{f}$ for any 2-cocycle $f$. As an application, we prove (Theorem 5.4) that if $g$ has a free $K$-base of $n$-elements, then $w$ - $\operatorname{dim}_{K} g_{f}=\operatorname{dim}_{K} g_{f}=n$ (where $w$-dim and dim denote the "algebra dimensions" of [1]).

The sixth section treats the case of abelian Lie algebras. The theorem of this section (Theorem 6.1) gives in particular a cohomological characterization of those $g_{f}$ which occur in quantum mechanics.

The appendix contains certain results on filtered modules and algebras. The first two propositions are probably well known. (They have been included since no explicit reference could be given.) The last proposition and its proof are due to Professor S. Eilenberg.

With a few exceptions where a change of notation is forced, we follow those of H. Cartan and S. Eilenberg as used in [1].

I would like to express here my deep indebtedness to Professor Eilenberg for his generous help, kind advice, and very useful criticism while this paper was in preparation. I am thankful to Professor K. Chandrasekharan for his constant encouragement and to the referee for his various helpful suggestions and improvements.

1. $f$-representations of a Lie algebra. Let $g$ be a Lie algebra over a commutative ring $K$ ( $K$ is assumed to have a unit element denoted by 1 ). Let $f$ be a 2-cocycle on the "standard complex" for $g[1$, p. 279] with values in $K$. We know that $f: \mathfrak{g} \times \mathfrak{g} \rightarrow K$ is a $K$-bilinear map which satisfies

$$
\begin{gathered}
f(x, x)=0 \quad \text { for every } x \in \mathfrak{g} . \\
f(x,[y, z])+f(y,[z, x])+f(z,[x, y])=0 \quad \text { for all } x, y, z \in \mathfrak{g} .
\end{gathered}
$$

Definition 1.1. An " $f$-representation" of $g$ over $K$ is a pair $(M, \phi)$ formed of a $K$-module $M$ and a $K$-linear map $\phi: \mathfrak{g} \rightarrow \operatorname{Hom}_{K}(M, M)$ such that for all $x, y \in g$,

$$
[[\phi(x), \phi(y)]]=\phi([x, y])+f(x, y) i_{M}
$$

(where $[[\alpha, \beta]]=\alpha \beta-\beta \alpha$ and $i_{M}$ is the identity map of $M$ ).

Proposition 1.2. Let $f: \mathfrak{g} \times \mathfrak{g} \rightarrow K$ be a set theoretic map. If $M$ is a faithful $K$-module and $\phi: \mathfrak{g} \rightarrow \operatorname{Hom}_{K}(M, M)$ a $K$-linear map such that (1.2) holds, then $f \in Z^{2}(g, K)$ (i.e., $f$ is a $K$-valued 2-cocycle on the standard complex).

Proof. Since $M$ is faithful as a $K$-module, we know that if $k \in K$ is such that $k i_{M}=0$, then $k=0$. In view of this remark, it is enough if we prove

$$
\begin{array}{rlrl}
\left(f\left(k x+k^{\prime} x^{\prime}, y\right)-k f(x, y)-k^{\prime} f\left(x^{\prime}, y\right)\right) i_{M} & =0, & & x, x^{\prime}, y \in \mathfrak{g}, k ; k \in K . \\
\left(f\left(x, k y+k^{\prime} y^{\prime}\right)-k f(x, y)-k^{\prime} f\left(x, y^{\prime}\right)\right) i_{M} & =0, & & x, y, y^{\prime} \in \mathfrak{g}, k ; k \in K . \\
f(x, x) i_{M} & =0, & x \in \mathfrak{g} . \\
(f(x,[y, z])+f(y,[z, x])+f(z,[x, y])) i_{M}=0, & & x, y, z \in \mathfrak{g} .
\end{array}
$$


The first two equations follow from the $K$-linearity of $\phi$ and the $K$ bilinearity of the bracket operation in $\mathrm{g}$. The third follows by putting $y=x$ in (1.2) and noting that $[x, x]=0$. We now prove the last assertion. (1.2) gives

$$
[[\phi(x), \phi([y, z])]]=\phi([x,[y, z]])+f(x,[y, z]) i_{M} .
$$

Applying (1.2) to the left hand side once again we see that it is equal to $[[\phi(x),[[\phi(y), \phi(z)]]]]$. We thus have

$$
[[\phi(x),[[\phi(y), \phi(z)]]]]=\phi([x,[y, z]])+f(x,[y, z]) i_{M} .
$$

We can write two more such equations permuting $x, y, z$ cyclically. Adding these equations, using the additivity of $\phi$ and the Jacobi identity, we get the required assertion.

REMARK 1.3. The above proposition shows that in some cases (for example if $K$ is a field) one need not explicitly assume in Definition 1.1 that $f \in Z^{2}(g, K)$, since it is actually implied by the definition.

Let $g$ be a Lie algebra and let $f \in Z^{2}(g, K)$. Consider the $K$-module $\mathfrak{g}^{\prime}=\mathfrak{g} \oplus K . z \quad(K . z$ being the free $K$-module generated by $z$ ) with the Lic algebra structure given by

$$
\left[(x, k z),\left(y, k^{\prime} z\right)\right]=([x, y], f(x, y) z), \quad x, y \in g, k ; \quad k^{\prime} \in K .
$$

$\mathfrak{g}^{\prime}$ is in fact the extension of $\mathfrak{g}$ by $K$ corresponding to the cocycle $f$. It is clear that any $f$-representation of $\mathfrak{g}$ is an ordinary representation of $\mathfrak{g}^{\prime}$ on which $z$ operates as the identity.

2. The algebra $g_{f}$. Let $g$ denote an arbitrary Lie algebra over the commutative ring $K$ and $f \in Z^{2}(\mathfrak{g}, K)$. Let $(M, \phi)$ be an $f$-representation. Let $T(\mathfrak{g})$ be the tensor algebra of the $K$-module $\mathfrak{g}$. The $K$-linear map $\phi$ can be uniquely extended to a $K$-algebra-homomorphism $\Phi: T(\mathfrak{g}) \rightarrow \operatorname{Hom}_{K}(M, M)$. The condition (1.2) implies that $\bar{\phi}$ vanishes on the two sided ideal $U_{f}(\mathfrak{g})$ generated by the elements

$$
x \otimes y-y \otimes x-[x, y]-f(x, y) ; \quad x, y \in \mathfrak{g} .
$$

Conversely, if $\phi: \mathfrak{g} \rightarrow \operatorname{Hom}_{K}(M, M)$ is any $K$-linear map such that $\phi$ vanishes on $U_{f}(\mathfrak{g})$, it is evident that $(M, \phi)$ is an $f$-representation.

Definition 2.1. The quotient of $T(\mathfrak{g})$ by the two sided ideal $U_{f}(\mathfrak{g})$ is called the "enveloping algebra of type $f$ " and denoted by $\mathfrak{g}_{f}$.

It is clear that there is a 1-1 correspondence between $f$-representations and left $g_{f}$-modules. This shows in particular that for a given $f \in Z^{2}(g, K)$ there always exists an $f$-representation-for instance $g_{f}$ itself.

REMARK 2.2. As with ordinary representations, we must distinguish between left and right $f$-representations. In particular, it should be noted that Definition 1.1 is more properly that of a left $f$-representation. We can give a similar definition of a right $f$-representation. $\mathfrak{g}_{-f}$ is a universal model for right representations. 
Let $\eta_{f}: T(\mathfrak{g}) \rightarrow \mathfrak{g} f$ denote the factorization map. The restriction of $\eta_{f}$ to $\mathfrak{g}$ yields a $K$-linear map $i_{f}: \mathfrak{g} \rightarrow \mathfrak{g}_{f}$ which satisfies for every $x, y \in \mathfrak{g}$

$$
\left[\left[i_{f}(x), i_{f}(y)\right]\right]=i_{f}([x, y])+f(x, y) .1 .
$$

Let $F_{p}\left(\mathfrak{g}_{f}\right)=\eta_{f}\left(\sum_{j \leq p} T_{j}(\mathfrak{g})\right)$. Then $\left(F_{p}\left(\mathfrak{g}_{f}\right)\right), p \geqq 0$, defines an increasing filtration of $\mathfrak{g}_{f}$ by $K$-submodules. Since $F_{p}\left(g_{f}\right) \cdot F_{q}\left(g_{f}\right) \subseteq F_{p+q}\left(g_{f}\right)$, we see $g_{f}$ is actually a filtered $K$-algebra. Let $E^{0}\left(\mathfrak{g}_{f}\right)$ denote as usual the graded algebra associated with $g_{f}$. Evidently $\eta_{f}$ induces a graded algebra homomorphism

$$
E^{0}\left(\eta_{f}\right): T(\mathfrak{g}) \rightarrow E^{0}\left(\mathfrak{g}_{f}\right) .
$$

Proposition 2.3. $E^{0}\left(\mathfrak{g}_{f}\right)$ is a commutative algebra.

Proof. In view of the fact that $1, i_{f}(\mathrm{~g})$ are a set of generators for the algebra $g_{f}$, the proposition is an immediate consequence of the

Lemma 2.4. For any sequence $a_{1}, \cdots, a_{p} \in g$ and any permutation $\sigma$ of $(1, \cdots, p)$ we have

$$
i_{f}\left(a_{1}\right) \cdots i_{f}\left(a_{p}\right)-i_{f}\left(a_{\sigma(1)}\right) \cdots i_{f}\left(a_{\sigma(p)}\right) \in F_{p-1}\left(g_{f}\right) .
$$

Proof. It is clearly sufficient to consider the case of a transposition interchanging two consecutive indices $j, j+1$. In this case the relation

$$
\left[\left[i_{f}\left(a_{j}\right), i_{f}\left(a_{j+1}\right)\right]\right]=i_{f}\left(\left[a_{j}, a_{j+1}\right]\right)+f\left(a_{j}, a_{j+1}\right) .1
$$

gives the required assertion.

Let $S(\mathfrak{g})$ denote the symmetric algebra on the $K$-module $\mathfrak{g}$. In view of Proposition 2.3, (2.3) yields a homomorphism

$$
\psi_{f}: S(\mathfrak{g}) \rightarrow E^{0}\left(\mathfrak{g}_{f}\right) \text {. }
$$

It is easy to see that $\psi_{f}$ is an epimorphism.

THEOREM 2.5. If $\mathrm{g}$ is $K$-free, $\psi_{f}$ is an isomorphism.

In order to prove the above theorem, we need another theorem which we now state and prove. Let $\left(x_{\alpha}\right)_{\alpha \in I}$ be a $K$-base for $\mathfrak{g}$. We assume that there is an order relation in $I$, which makes it a totally ordered set. Let $y_{\alpha}=i_{f}\left(x_{\alpha}\right)$. If $J=\left(\alpha_{1}, \cdots, \alpha_{p}\right)$ is a finite sequence of elements of $I$, we write $y_{J}=y_{\alpha_{1}}, \cdots$, $y_{\alpha_{p}}$ and call $p$ the length of $J$. We say that $J$ is increasing if $\alpha_{1} \leqq \cdots \leqq \alpha_{p}$. We set $y_{J}=1$ if $J$ is empty and regard the empty set increasing. The theorem we intend to prove is the following:

TheOREM 2.6 (PoINCARÉ-WitT). The elements $y_{J}$ corresponding to finite increasing sequences $J$ form a $K$-base for $\mathfrak{g}_{f}$.

Proof $\left({ }^{2}\right)$. If $\mathfrak{g}^{\prime}$ is defined as in $\$ 1$, the following lemma can be easily checked.

(2) I am grateful to the referee for pointing out this simple proof. 
Lemma 2.7. If $\mathfrak{g}$ is $K$-free, the mapping $\mathfrak{g}^{\prime} \rightarrow T(\mathfrak{g})$ given by $(x, k z) \rightarrow x+k$ induces an isomorphism $\mathfrak{g}_{0}^{\prime} /(z-1) \approx \mathfrak{g}_{f}\left((z-1)\right.$ being the 2-sided ideal of $\mathfrak{g}_{0}^{\prime}$ generated by $z-1$ ).

It is obvious that $\left\{\left(x_{\alpha}, 0\right)\right\}_{\alpha \in I},(0, z)$ form a $K$-basis for $g^{\prime}$. If we then take the $K$-basis of $g_{0}^{\prime}$ constructed in the usual manner from these elements $\left[1\right.$, p. 271], it is easy to see that the $y_{j}^{s}$ are precisely their images under the isomorphism given in Lemma 2.7. This proves Theorem 2.6.

Corollary 2.8. If $\mathfrak{g}$ is $K$-free, $i_{f}: \mathfrak{g} \rightarrow \mathfrak{g}_{f}$ is a $K$-monomorphism.

Proof. Theorem 2.6 implies in particular that the elements $y_{\alpha}=i_{f}\left(x_{\alpha}\right)$ are $K$-linearly independent and this proves the corollary.

If $J=\left(\alpha_{1}, \cdots, \alpha_{p}\right)$, we set $x_{J}=x_{\alpha_{1}} \cdots x_{\alpha_{p}}$ in $S(\mathfrak{g})$.

Proof of Theorem 2.5. In view of Theorem 2.6, there exists a unique $K$ linear map $\psi^{\prime}: g_{f} \rightarrow S(\mathfrak{g})$ which satisfies for any increasing $J$, the condition $\psi^{\prime}\left(y_{J}\right)=x_{J}$. If we assume on $S(\mathrm{~g})$ the evident filtration, it is clear that $\psi^{\prime}$ is a mapping of filtered $K$-modules. There is thus an induced mapping $E^{0}\left(\psi^{\prime}\right): E^{0}\left(\mathfrak{g}_{f}\right) \rightarrow S(\mathfrak{g})$. It is clear that $E^{0}\left(\psi^{\prime}\right)$ is inverse to $\psi_{f}$. Hence $\psi_{f}$ is bijective and therefore an isomorphism.

Corollary 2.9. If $K$ is Noetherian and $\mathfrak{g}$ is $K$-free on a finite base, $\mathfrak{g}_{f}$ is (both left and right) Noetherian.

Proof. Under our assumptions it is well known that $S(\mathfrak{g})$ is Noetherian. Using Theorem 2.5 and the known theorem that a filtered algebra is Noetherian if its associated graded algebra is so, the corollary is clear.

3. Classification of filtered algebras. In this section, by a filtered $K$ algebra, we shall always mean one with an increasing filtration $F$ such that $F_{q}=0$ for $q<0$ and $F_{0}=K$.

Let $S=\sum_{p \geq 0} S_{p}$ be a fixed graded $K$-algebra. Let us consider the family of all pairs $\left(\Lambda, \psi_{\Lambda}\right), \Lambda$ being a filtered $K$-algebra and $\psi_{\Lambda}: S \rightarrow E^{0}(\Lambda)$ a graded $K$-algebra isomorphism (where $E^{0}(\Lambda)$ denotes as usual the graded $K$-algebra associated with the filtered algebra $\Lambda)$. A map $\theta:\left(\Lambda, \psi_{\Lambda}\right) \rightarrow\left(\Gamma, \psi_{\Gamma}\right)$ is a filtered $K$-algebra homomorphism $\theta: \Lambda \rightarrow \Gamma$ such that if $E^{0}(\theta): E^{0}(\Lambda) \rightarrow E^{0}(\Gamma)$ is the graded algebra homomorphism induced by $\theta$, the diagram

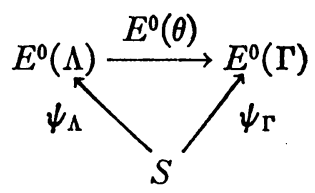

is commutative. Composition of maps is defined in the obvious way. The resulting category is denoted by $A(S)$.

If $\theta:\left(\Lambda, \psi_{\Lambda}\right) \rightarrow\left(\Gamma, \psi_{\Gamma}\right)$, then $E^{0}(\theta): E^{0}(\Lambda) \rightarrow E^{0}(\Gamma)$ is an isomorphism, because $E^{0}(\theta)=\psi_{\Gamma} \circ \psi_{\Lambda}^{-1}$. Then by Proposition 1 of the appendix, $\theta$ is bijective, $\theta^{-1}$ is compatible with the filtrations and $\theta^{-1}:\left(\Gamma, \psi_{\Gamma}\right) \rightarrow\left(\Lambda, \psi_{\Lambda}\right)$ is a map in 
$A(S)$. Thus every map in $A(S)$ is an isomorphism; i.e. $A(S)$ is a groupoid.

Now assume that $S$ is commutative. Given $\left(\Lambda, \psi_{\Lambda}\right)$ we have $\left[\left[F_{1} \Lambda, F_{1} \Lambda\right]\right]$ $\subset F_{2} \Lambda$. Since $E^{0}(\Lambda)$ must be commutative, it follows that $\left[\left[F_{1} \Lambda, F_{1} \Lambda\right]\right] \subset F_{1} \Lambda$ so that $F_{1} \Lambda$ acquires the structure of a Lie algebra. It is clear that $K=F_{0} \Lambda$ is an ideal of $F_{1} \Lambda$. Thus, the isomorphism $S_{1} \approx F_{1} \Lambda / F_{0} \Lambda$ (given by $\psi_{\Lambda}$ ) induces a Lie algebra structure on $S_{1}$ which we shall denote by $\mathfrak{g}$. The same isomorphism yields an exact sequence

$$
0 \rightarrow K \rightarrow F_{1} \Lambda \stackrel{\phi_{\Lambda}}{\rightarrow} g \rightarrow 0
$$

which is an extension of Lie algebras. Since $K$ is in the centre of $F_{1} \Lambda$, this is an abelian extension; i.e. an extension corresponding to the trivial representation of $\mathfrak{g}$ in $K$.

Now assume that $S_{1}$ is $K$-projective. There exists then a $K$-homomorphism $t: \mathfrak{g} \rightarrow F_{1} \Lambda$ such that $\phi_{\Lambda} \circ t=$ identity. We then have the well-known relation

$$
[[t(x), t(y)]]=t([x, y])+f(x, y) ; x, y, \in \mathfrak{g} .
$$

It is known that $f$ is a 2 -cocycle on the standard complex of $g$ and that the cohomology class $f$ of $f$ is independent of the choice of $t$.

Thus, we see that every object $\left(\Lambda, \psi_{\Lambda}\right)$ in $A(S)$, for $S$ commutative and $S_{1} K$-projective, determines a pair $(\mathfrak{g}, \boldsymbol{f})$ where $\mathfrak{g}$ is a Lie algebra structure on $S_{1}$ and $f \in H^{2}(\mathfrak{g}, K)$. Isomorphic objects in $A(S)$ determine the same pair.

THeOREm 3.1. Let $L$ be a free $K$-module and $S(L)$ the symmetric $K$-algebra on L. The isomorphism classes of objects in $A(S(L))$ are then in a 1-1 correspondence with pairs $(\mathfrak{g}, \boldsymbol{f})$ where $\mathfrak{g}$ is a Lie algebra structure on $L$ and $f$ is an element in $H^{2}(g, K)$. If $f$ is a cocycle in the class $f$, then $\left(g_{f}, \psi_{f}\right)$ is an object in the isomorphism class determined by $(\mathrm{g}, \mathrm{f})$.

Proof. Let $\mathfrak{g}$ be a Lie algebra structure on $L$ and let $f$ be a cocycle in a cohomology class $f \in H^{2}(\mathfrak{g}, K)$. Then, by Theorem $2.5,\left(\mathfrak{g}_{f}, \psi_{f}\right)$ is an object in $A(S(L))$. Consider the exact sequence

$$
0 \rightarrow K \rightarrow F_{1}\left(\mathfrak{g}_{f}\right) \stackrel{\phi_{f}}{\rightarrow} \mathfrak{g} \rightarrow 0
$$

where $\phi_{f}$ is induced by $\psi_{f}$. The map $i_{f}: g \rightarrow F_{1}\left(g_{f}\right)$ is a $K$-linear section and the relation (2.2) shows that $\left(g_{f}, \psi_{f}\right)$ yields $(g, f)$.

Let $\left(\Lambda, \psi_{\Lambda}\right)$ be another object in $A(S(L))$ yielding $(\mathfrak{g}, f)$. Choose $t: \mathfrak{g} \rightarrow F_{1} \Lambda$ so that (3.2) is valid for the cocycle $f$. Let $\bar{t}: T(\mathfrak{g}) \rightarrow \Lambda$ be the natural extension of $t$. If $x, y \in \mathfrak{g}$, then,

$$
\bar{t}(x \otimes y-y \otimes x-[x, y]-f(x, y))=[[t(x), t(y)]]-t([x, y])-f(x, y)=0 .
$$

Thus $\bar{t}\left(U_{f}(\mathfrak{g})\right)=(0)$, so that $\bar{t}$ induces a homomorphism

$$
t^{\sharp}: \mathfrak{g}_{f} \rightarrow \Lambda
$$


of filtered algebras. Further, we have the diagram

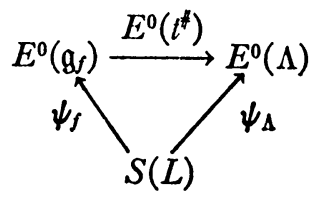

For $x \in L$, we know that $t(x)$ is in the coset $\psi_{\Lambda}(x)$ of $F_{1} \Lambda \bmod F_{0} \Lambda$. Thus,

$$
E^{0}\left(t^{*}\right) \psi_{f}(x)=E^{0}\left(t^{\sharp}\right) i_{f}(x)=\psi_{\Lambda}(x) .
$$

Hence the diagram above is commutative. Thus, $t^{t}:\left(g_{f}, \psi_{f}\right) \rightarrow\left(\Lambda, \psi_{\Lambda}\right)$ is a map and therefore an isomorphism.

It follows in particular from Theorem 3.1 that $\left(g_{f}, \psi_{f}\right)$ and $\left(g_{f^{\prime}}, \psi_{f^{\prime}}\right)$ are isomorphic, if and only if, $f$ and $f^{\prime}$ are cohomologous. The following proposition is a more explicit formulation of this result.

Proposition 3.2. Let $\mathfrak{g}$ be a $K$-free Lie algebra and let $f, f^{\prime} \in Z^{2}(\mathfrak{g}, K)$ be cohomologous cocycles. The maps $\theta:\left(\mathfrak{g}_{f}, \psi_{f}\right) \rightarrow\left(\mathfrak{g}_{f^{\prime}}, \psi_{f^{\prime}}\right)$ are then in 1-1 correspondence with the 1-cochains $h \in C^{1}(\mathfrak{g}, K)$ such that $f^{\prime}-f=\delta h$. The correspondence is defined implicitly by the relation

$$
\theta_{h}\left(i_{f}(x)\right)=h(x)+i_{f^{\prime}}(x), \quad x \in \mathfrak{g} .
$$

Moreover, if $f, f^{\prime}, f^{\prime \prime} \in Z^{2}(g, K)$ are such that $f^{\prime}-f=\delta h$ and $f^{\prime \prime}-f^{\prime}=\delta h^{\prime}$; $h, h^{\prime} \in C^{1}(\mathrm{~g}, K)$, then $\theta_{h+h^{\prime}}=\theta_{h^{\prime}} \circ \theta_{h}$.

Proof. The relation $f^{\prime}-f=\delta h$ signifies that

$$
f(x, y)-f^{\prime}(x, y)=h([x, y])
$$

for all $x, y \in \mathfrak{g}$. It is then easily seen that the $K$-algebra automorphism of $T(\mathfrak{g})$ which maps $x$ into $x+h(x)$ takes the ideal $U_{f}(\mathfrak{g})$ on $U_{f^{\prime}}(\mathfrak{g})$. There is thus an induced $K$-algebra isomorphism $\mathfrak{g}_{f} \rightarrow \mathfrak{g}_{f^{\prime}}$ and it is clear that (3.3) is satisfied and that it is a map in $A(S(L))$. Conversely suppose that $\theta:\left(g_{f}, \psi_{f}\right) \rightarrow\left(g_{f^{\prime}}, \psi_{f^{\prime}}\right)$ is any map. For any $x \in \mathfrak{g}$, we have

$$
\theta\left(i_{f}(x)\right)=h(x)+i_{f^{\prime}}(x)
$$

where $h(x) \in K$. It is clear that $h: \mathfrak{g} \rightarrow K$ is well defined. Using the above and the relation (2.2) for both $f$ and $f^{\prime}$, it is evident that (3.4) holds. The other assertion of the proposition is clear.

CoRollary 3.3. Let $\mathrm{g}$ be a $K$-free Lie algebra and let $f \in Z^{2}(\mathrm{~g}, K)$. There is an isomorphism between the group of maps $\left(\mathfrak{g}_{f}, \psi_{f}\right) \rightarrow\left(\mathfrak{g}_{f}, \psi_{f}\right)$ and the group $H^{1}(\mathfrak{g}, K)$. The correspondence is defined by the relation

$$
\theta\left(i_{f}(x)\right)=h(x)+i_{f}(x)
$$

for $\theta:\left(\mathfrak{g}_{f}, \psi_{f}\right) \rightarrow\left(\mathfrak{g}_{f}, \psi_{f}\right)$ and $h \in H^{1}(\mathfrak{g}, K)=Z^{1}(\mathfrak{g}, K)$. 
REMARK 3.4. It should be noted that $g_{f}$ and $g_{f^{\prime}}$ may be isomorphic as filtered algebras without $\left(g_{f}, \psi_{f}\right)$ and $\left(g_{f^{\prime}}, \psi_{f^{\prime}}\right)$ being isomorphic, i.e. without $f$ and $f^{\prime}$ being cohomologous. This is the case for instance in the following example. Let $g$ be the abelian Lie algebra over a field $K$ with a $K$-base consisting of two elements $y, z$. In this case, it is clear that $B^{2}(g, K)=(0)$ and the mapping $Z^{2}(\mathrm{~g}, K) \rightarrow K$ given by $f \rightarrow f(y, z)=k \in K$ is an isomorphism of $K$ modules. Thus $\mathfrak{g}_{k}=g_{f}$ in this case is the algebra on two generators with the relation $[[y, z]]=k \in K$. The case $k=0$ leads to the polynomial algebra in $y, z$. If $k \neq 0$, then algebras $g_{k}$ and $g_{1}$ are isomorphic as filtered algebras. Denoting for the moment the generators of the first algebra by $y^{\prime}, z^{\prime}$ and the second by $y, z$, the required isomorphism $\mathrm{g}_{k} \rightarrow \mathrm{g}_{1}$ is given by $y^{\prime} \rightarrow y, z^{\prime} \rightarrow k z$. This map clearly preserves filtration, but is not a map in the category $A(K[y, z])$ unless $k=1$.

REMARK 3.5. If $g$ is a semi-simple Lie algebra over a field $K$ of characteristic zero, it is known [2, p. 113, Th. 21.1] that $H^{1}(\mathfrak{g}, K)=H^{2}(\mathfrak{g}, K)=(0)$. An application of Theorem 3.1 shows that for any $f \in Z^{2}(\mathfrak{g}, K)$, we have $\left(g_{f}, \psi_{f}\right) \approx\left(g_{0}, \psi_{0}\right)$. ( $g_{0}$ is the usual enveloping algebra of $g$.) Moreover, Corollary 3.3 shows that the only map of $\left(g_{f}, \psi_{f}\right)$ into itself is the identity.

4. Some homological properties of a Lie algebra. In this section we shall compute certain of the usual homology and cohomology $(K-)$ modules of a Lie algebra $g$ with a finite $K$-base. This amounts to a study of $\mathfrak{g}_{f}$ for $f=0$. These computations will be used in the next section to obtain information about the homological structure of $g_{f}$ for any $f \in Z^{2}(g, K)$.

We begin by recalling the definition of the homology and cohomology of any Lie algebra $\mathfrak{g}$. We know that there is a $K$-algebra epimorphism,

$$
\epsilon: g_{0} \rightarrow K
$$

defined by setting $\epsilon(x)=(0)$ for all $x \in \mathrm{g}$. Let $I=$ kernel of $\epsilon$. We regard $K$ as a left $\mathfrak{g}_{0}$-module through $\epsilon$. For any right representation $A$ of $\mathfrak{g}$ and a left representation $C$ of $\mathfrak{g}$, the homology and cohomology modules of $\mathfrak{g}$ with coefficients respectively in $A$ and $C$ are defined, for $n \geqq 0$, by

$$
\begin{aligned}
& H_{n}(\mathfrak{g}, A)=\operatorname{Tor}_{n}^{\mathfrak{g}_{0}}(A, K), \\
& H^{n}(\mathfrak{g}, C)=\operatorname{Ext}_{\mathfrak{g}_{0}}^{n}(K, C) .
\end{aligned}
$$

The modules on the right hand side can be computed using any left $g_{0}$ projective resolution of $K$. If $\mathfrak{g}$ is assumed to be $K$-free, we can use in particular the "Standard complex" $X=\left(X_{i}, d_{i}\right), i \geqq 0$, for $\mathfrak{g}$, where $X_{i}=g_{0} \otimes_{K} E_{i}(\mathfrak{g})$, $\left(E_{i}(g)\right.$ denoting the $i$ th homogeneous component of the exterior algebra $E(\mathfrak{g})$ on $\mathfrak{g})$ and $d_{i}: X_{i} \rightarrow X_{i-1}$ is the $g_{0}$-homomorphism given by

$$
\begin{aligned}
d_{i}\left(1 \otimes\left(x_{1} \cdots x_{i}\right)\right)= & \sum_{1 \leq j \leq i}(-1)^{j+1} x_{j} \otimes\left(x_{1} \cdots \hat{x}_{j} \cdots x_{i}\right) \\
& +\sum_{1 \leq j<k \leq i}(-1)^{j+k} 1 \otimes\left(\left[x_{j}, x_{k}\right] x_{1} \cdots \hat{x}_{j} \cdots \hat{x}_{k} \cdots x_{i}\right) .
\end{aligned}
$$


From now on, let $\mathfrak{g}$ denote a Lie algebra with a free $K$-base $x_{1}, \cdots, x_{n}$ and let $\left[x_{i}, x_{j}\right]=\sum_{1 \leq k \leq n} C_{i j}^{k} x_{k}, C_{i j}^{k} \in K$. In this case $X$ has length $n$ since $E_{i}(\mathfrak{g})=(0)$ for $i>n$. In particular, we deduce that

$$
H_{r}(\mathfrak{g}, A)=H^{r}(\mathfrak{g}, C)=(0) \quad \text { for } r>n .
$$

We propose to compute $H_{n}(\mathfrak{g}, A)$ and $H^{n}(\mathfrak{g}, C)$. For this purpose, we first simplify the formula for $d_{n}$. Set $\omega=1 \otimes x_{1} \cdots x_{n}$ and $\omega_{i}=1 \otimes x_{1} \cdots \hat{x}_{i} \cdots x_{n}$, $1 \leqq i \leqq n$. Since

$$
\begin{aligned}
1 \otimes\left(\left[x_{i}, x_{j}\right] x_{1} \cdots \hat{x}_{i} \cdots \hat{x}_{j} \cdots x_{n}\right) & =\sum_{k} 1 \otimes C_{i j}^{k}\left(x_{k} x_{1} \cdots \hat{x}_{i} \cdots \hat{x}_{j} \cdots x_{n}\right) \\
& =(-1)^{i+1} C_{i j}^{i} \omega_{j}-(-1)^{j+1} C_{i j}^{j} \omega_{i},
\end{aligned}
$$

it follows that

$$
\begin{aligned}
\sum_{1 \leq i<j \leqslant n}(-1)^{i+j} 1 & \otimes\left(\left[x_{i}, x_{j}\right] x_{1} \cdots \hat{x}_{i} \cdots \hat{x}_{j} \cdots x_{n}\right) \\
& =\sum_{1 \leq i<j \leq n}(-1)^{j+1} C_{i j \omega_{j}}^{i}-\sum_{1 \leq i<j \leq n}(-1)^{i+1} C_{i j \omega_{i}}^{j}=\sum_{i, j}(-1)^{i} C_{i j \omega_{i}}^{j} .
\end{aligned}
$$

(4.3) now gives

$$
d_{n}(\omega)=\sum_{1 \leq i \leq n}(-1)^{i+1}\left(x_{i}-\sum_{j} C_{i j}^{j}\right) \omega_{i} .
$$

The $K$-linear mapping Ad: $\mathfrak{g} \rightarrow \operatorname{Hom}_{K}(\mathfrak{g}, \mathfrak{g})$, defined as usual by $(\operatorname{Ad} x)(y)$ $=[x, y]$, evidently satisfies

$$
\operatorname{Ad}([x, y])=[[\operatorname{Ad} x, \operatorname{Ad} y]] .
$$

If we define

$$
h: \mathfrak{g} \rightarrow K
$$

by $h(x)=$ Trace $(\operatorname{Ad} x), h$ is a $K$-linear map which satisfies $h\left(x_{i}\right)=\sum_{j} C_{i j}^{j}$ $1 \leqq i \leqq n$. In view of (4.6), it is also clear that for all $x, y \in \mathfrak{g}, h([x, y])=0$ and hence that $h$ is a 1-cocycle on $X$. Corollary 3.3 now shows that there exist $K$-algebra automorphisms $\alpha$ and $\beta$ of $\mathfrak{g}_{0}$ such that for all $x \in \mathfrak{g}$,

$$
\begin{aligned}
& \alpha(x)=x+h(x), \\
& \beta(x)=x-h(x) .
\end{aligned}
$$

Clearly, $\alpha^{-1}=\beta$.

(4.5) now takes the form

$$
d_{n}(\omega)=\sum_{1 \leqq i \leqq n}(-1)^{i+1} \beta\left(x_{i}\right) \omega_{i} .
$$


Now, $H_{n}(\mathfrak{g}, A)=H_{n}\left(A \otimes \mathfrak{g}_{0} X\right)=\operatorname{ker}\left(i_{A} \otimes_{\mathfrak{g}_{0}} d_{n}\right)$, where $i_{A}$ denotes the identity map of $A$. Using this and the formula (4.8) for $d_{n}$, it follows that

$$
\begin{aligned}
H_{n}(\mathfrak{g}, A) & =\left\{a \otimes \omega \in A \otimes X_{n} \mid a \beta\left(x_{i}\right)=0,1 \leqq i \leqq n\right\} \\
& =\{a \otimes \omega \mid a \beta(I)=0\} .
\end{aligned}
$$

Thus the $K$-isomorphism $A \otimes X_{n} \approx A$ given by $a \otimes \omega \rightarrow a$ induces an isomorphism

$$
H_{n}(\mathfrak{g}, A) \approx \text { annihilator of } \beta(I) \text { in } A .
$$

On the other hand, $H^{n}(\mathfrak{g}, C)=H_{n}\left(\operatorname{Hom}_{\mathfrak{g}_{0}}\left(X_{n}, C\right)\right)=\operatorname{Hom}_{\mathfrak{g}_{0}}\left(X_{n}, C\right) / B^{n}(\mathfrak{g}, C)$, where $B^{n}(\mathfrak{g}, C)=$ im $\operatorname{Hom}_{\mathbb{B}_{0}}\left(d_{n}, i_{C}\right)\left(i_{C}\right.$ denoting the identity map of $\left.C\right)$. Using (4.8) we get

$$
\begin{array}{r}
B^{n}(\mathfrak{g}, C)=\left\{g \in \operatorname{Hom}_{\mathfrak{g} 0}\left(X_{n}, C\right) \mid g(\omega)=\sum_{i}(-1)^{i+1} \beta\left(x_{i}\right) \lambda\left(\omega_{i}\right)\right. \\
\text { for some } \left.\lambda \in \operatorname{Hom}_{\mathfrak{g}_{0}}\left(X_{n-1}, C\right)\right\} .
\end{array}
$$

It is then clear that the $K$-isomorphism $\operatorname{Hom}_{\mathbb{g}_{0}}\left(X_{n}, C\right) \approx C$ given by $g \rightarrow g(\omega)$ induces an isomorphism

$$
H^{n}(\mathfrak{g}, C) \approx C / \beta(I) C .
$$

Combining (4.4), (4.9) and (4.10), we obtain the following

THEOREM 4.1. Let $\mathrm{g}$ be a Lie algebra with a free $K$-base of $n$-elements. If $A$ is any right representation of $\mathfrak{g}$ and $C$ any left representation of $\mathfrak{g}$, we have

$$
\begin{aligned}
H_{n}(\mathfrak{g}, A) \approx \text { annihilator of } \beta(I) \text { in } A, H_{r}(\mathfrak{g}, A) & =(0) & \text { for } r>n . \\
H^{n}(\mathfrak{g}, C) \approx C / \beta(I) C, & H^{r}(\mathfrak{g}, C)=(0) & \text { for } r>n .
\end{aligned}
$$

We denote by $A_{\alpha}$ (resp. ${ }_{\alpha} C$ ), $A$ (resp. $C$ ) considered as a $g_{0}$-module through $\alpha$. With this notation, the above theorem has the following immediate

CoROllary 4.2. If $\mathfrak{g}$ has a free $K$-base of $n$-elements, we have

$$
\begin{aligned}
& H_{n}\left(\mathfrak{g}, A_{\alpha}\right) \approx \text { annihilator of } I \text { in } A . \\
& H^{n}\left(\mathfrak{g},{ }_{\alpha} C\right) \approx C / I C .
\end{aligned}
$$

5. Homology and cohomology of $g_{f}$. We begin this section by recalling briefly the definition of homology and cohomology of associative algebras.

Let $\Lambda$ be a $K$-algebra and $A$ any two sided $\Lambda$-module. $A$ can then be considered as a left (or right) $\Lambda \otimes_{K} \Lambda^{*}=\Lambda^{e}$-module, by setting $\left(\mu \otimes \nu^{*}\right) a=\mu a \nu$ (or $\left.a\left(\mu \otimes \nu^{*}\right)=\nu a \mu\right) ; \mu, \nu \in \Lambda, a \in A . \Lambda$ itself being a two sided $\Lambda$-module, it is thus a left $\Lambda^{e}$-module with operators $\left(\mu \otimes \nu^{*}\right) \lambda=\mu \lambda \nu$. In particular, taking $\lambda=1$, we obtain a mapping

$$
\rho: \Lambda^{e} \rightarrow \Lambda
$$


given by $\rho\left(\mu \otimes \nu^{*}\right)=\mu \nu$. Clearly $\rho$ is an epimorphism of left $\Lambda^{e}$-modules. The $n$th homology and cohomology groups of $\Lambda$ with coefficients in $A$ are defined by

$$
\begin{aligned}
& H_{n}(\Lambda, A)=\operatorname{Tor}_{n}^{\Lambda^{\bullet}}(A, \Lambda)\left(A_{\left.\Lambda^{\bullet}, \Lambda^{\bullet} \Lambda\right) .}\right. \\
& H^{n}(\Lambda, A)=\operatorname{Ext}_{\Lambda^{\bullet}}^{n}(\Lambda, A)\left(\Lambda^{\bullet} \Lambda, \Lambda^{\bullet} A\right) .
\end{aligned}
$$

We define as usual the "weak" and the cohomological dimensions of $\Lambda$ denoted respectively by $w$ - $\operatorname{dim}_{K} \Lambda$ and $\operatorname{dim}_{K}-\Lambda$, as follows:

$w$-dim $\operatorname{di}_{K} \Lambda=$ largest integer $n$, if it exists, for which there exists a two sided $\Lambda$-module $A$ with $H_{n}(\Lambda, A) \neq(0)$, .

$$
=\infty \text {, if no such integer exists. }
$$

$\operatorname{dim}_{K}-\Lambda=$ largest integer $n$, if it exists, for which there exists a two sided $\Lambda$-module $A$ with $H^{n}(\Lambda, A) \neq(0)$,

$$
=\infty \text {, if no such integer exists. }
$$

Let now $g$ be a Lie algebra over $K$. We set $\Lambda_{f}=g_{f}^{e}=g_{f} \otimes_{K} g_{f}^{*}$ and let $J$ denote the kernel of the map $\rho: \Lambda_{f} \rightarrow g_{f}$ of (5.1).

The map $D: g \rightarrow \Lambda_{f}$, defined for $x \in g$ by

$$
D(x)=i_{f}(x) \otimes 1-1 \otimes\left(i_{f}(x)\right)^{*},
$$

is evidently $K$-linear and hence can be extended to a $K$-algebra-homomorphism $D: T(g) \rightarrow \Lambda_{f}$. If $x, y \in g$ we have

$$
\begin{aligned}
D(x \otimes y-y \otimes x-[x, y])= & \left(i_{f}(x) \otimes 1-1 \otimes i_{f}(x)^{*}\right)\left(i_{f}(y) \otimes 1-1 \otimes i_{f}(y)^{*}\right) \\
& -\left(i_{f}(y) \otimes 1-1 \otimes i_{f}(y)^{*}\right)\left(i_{f}(x) \otimes 1-1 \otimes i_{f}(x)^{*}\right) \\
& -\left(i_{f}([x, y]) \otimes 1-1 \otimes i_{f}([x, y])^{*}\right) \\
= & \left(\left[\left[i_{f}(x), i_{f}(y)\right]\right]-i_{f}([x, y])\right) \otimes 1 \\
& -1 \otimes\left(\left[\left[i_{f}(x), i_{f}(y)\right]\right]-i_{f}([x, y])\right)^{*} \\
= & f(x, y) \otimes 1-1 \otimes f(x, y) . \\
= & 0 .
\end{aligned}
$$

Hence $D$ induces a $K$-algebra homomorphism

$$
D: g_{0} \rightarrow \Lambda_{f}
$$

If $A$ is any two sided $g_{f}$-module, we denote by ${ }_{D} A$ (or $A_{D}$ ), $A$ considered as a left (or right) $g_{0}$-module.

LEMMA 5.1. $\Lambda_{f} D(I)=J$.

Proof. By [1, p. 168, Proposition 3.1], $J$ is the left ideal generated by the elements $u \otimes 1-1 \otimes u^{*}$ for $u \in g_{f}$. In view of the relation $u v \otimes 1-1 \otimes(u v)^{*}=(u \otimes 1)\left(v \otimes 1-1 \otimes v^{*}\right)+\left(1 \otimes v^{*}\right)\left(u \otimes 1-1 \otimes u^{*}\right)$, 
valid for $u, v \in \mathfrak{g}_{f}$, we find that $J$ is the left ideal of $\Lambda_{f}$ generated by the elements $i_{f}(x) \otimes 1-1 \otimes i_{f}(x)^{*}=D(x)$. Since the elements $x \in \mathfrak{g}$ generate $I$ in $g_{0}$, the required assertion is clear.

Proposition 5.2. If $\mathrm{g}$ is $K$-free, $\left(\Lambda_{f}\right)_{D}$ is a free $\mathrm{g}_{0}$-module.

Proof. Since $g_{f}$ and $g_{f}^{*}$ are filtered algebras, there is a natural filtration on $\Lambda_{f}=\mathfrak{g}_{f} \otimes_{K} \mathfrak{g}_{f}^{*}$. Proposition 7 of the appendix gives an isomorphism $E^{0}\left(g_{f}\right) \otimes_{K} E^{0}\left(g_{f}^{*}\right) \approx E^{0}\left(\Lambda_{f}\right)$. Combining this with the evident identification $E^{0}\left(\mathfrak{g}_{f}^{*}\right)=E^{0}\left(\mathfrak{g}_{f}\right)$, we get an isomorphism

$$
E^{0}\left(\mathfrak{g}_{f}\right) \otimes E^{0}\left(\mathfrak{g}_{f}\right) \approx E^{0}\left(\Lambda_{f}\right) .
$$

In what follows, we regard the above isomorphism as an identification. It is clear that $D: g_{0} \rightarrow \Lambda_{f}$ preserves filtration. Thus, there is an induced graded algebra homomorphism

$$
E^{0}(D): E^{0}\left(\mathfrak{g}_{0}\right) \rightarrow E^{0}\left(\mathfrak{g}_{f}\right) \otimes E^{0}\left(\mathfrak{g}_{f}\right) .
$$

By Proposition 2 of the appendix, it suffices to show that $\left(E^{0}\left(g_{f}\right) \otimes E^{0}\left(g_{f}\right)\right)_{E^{0}(D)}$ is $E^{0}\left(g_{0}\right)$-free in the graded sense. Now Theorem 2.5 shows that the maps $\psi_{0}: S(\mathfrak{g}) \rightarrow E^{0}\left(g_{0}\right)$ and $\psi_{f}: S(\mathfrak{g}) \rightarrow E^{0}\left(g_{f}\right)$ are isomorphisms. Since the diagram

$$
\begin{gathered}
\mathrm{S}(\mathfrak{g}) \stackrel{F}{\longrightarrow} S(\mathfrak{g}) \otimes S(\mathfrak{g}) \\
\downarrow \psi_{0} \\
E^{0}\left(\mathfrak{g}_{0}\right) \stackrel{E^{0}(D)}{\downarrow} E^{0}\left(\mathfrak{g}_{f}\right) \otimes \psi_{f}\left(\mathfrak{g}_{f}\right),
\end{gathered}
$$

where $F(x)=x \otimes 1-1 \otimes x$, is clearly commutative, it suffices to show that $(S(\mathfrak{g}) \otimes S(\mathfrak{g}))_{F}$ is $S(\mathfrak{g})$-free. Consider now the automorphism $\gamma$ of $S(\mathfrak{g}) \otimes S(\mathfrak{g})$ given by $\gamma(x \otimes 1)=x \otimes 1, \gamma(1 \otimes x)=x \otimes 1-1 \otimes x$. Then, $\gamma F(x)=1 \otimes x$. It is enough to show that $(S(\mathrm{~g}) \otimes S(\mathrm{~g}))_{\gamma F}$ is $S(\mathrm{~g})$-free. This is however clear, since, if $\left\{t_{\beta}\right\}$ is a $K$-base for $S(\mathfrak{g})$, then $\left\{t_{\beta} \otimes 1\right\}$ is an $S(\mathrm{~g})$-base for $(S(\mathrm{~g}) \otimes S(\mathrm{~g}))_{\gamma}$.

Proposition 5.3. Let $\mathrm{g}$ be $K$-free. Then, if $X$ is a left $\mathrm{g}_{0}$-projective resolution of $K,\left(\Lambda_{f}\right)_{D} \otimes g_{0} X$ is a left $\Lambda_{f}$-projective resolution of $g_{f}$. Moreover, for any two sided $\mathfrak{g}_{f}$-module $A$ and any $n \geqq 0$, we have the isomorphisms,

$$
\begin{aligned}
& H_{n}\left(\mathrm{~g}_{f}, A\right) \approx H_{n}\left(\mathfrak{g}, A_{D}\right), \\
& H^{n}\left(\mathfrak{g}_{f}, A\right) \approx H^{n}\left(\mathfrak{g}, D_{D} A\right) .
\end{aligned}
$$

Proof. Since $X$ is $g_{0}$-projective and $\Lambda_{f}$ is $\Lambda_{f}$-projective, it follows that $\left(\Lambda_{f}\right)_{D} \otimes_{g_{0}} X$ is $\Lambda_{f}$-projective. Also, for $i>0, H_{i}\left(\left(\Lambda_{f}\right)_{D} \otimes_{g_{0}} X\right)=\operatorname{Tor}_{i}^{g_{0}}\left(\left(\Lambda_{f}\right)_{D}, K\right)$ $=(0)$, since, by Proposition 5.2, $\left(\Lambda_{f}\right)_{D}$ is $g_{0}$-free. Thus $\left(\Lambda_{f}\right)_{D} \otimes_{\mathfrak{g}_{0}} X$ is a $\Lambda_{f}$ projective resolution of $\left(\Lambda_{f}\right)_{D} \otimes_{\mathfrak{g}_{0}} K$. To finish the first part of the proposition we need only show that there is a left $\Lambda_{f}$-isomorphism $\left(\Lambda_{f}\right)_{D} \otimes_{\mathfrak{g}_{0}} K \approx \mathfrak{g}_{f}$. To do this, consider the exact sequence of $g_{0}$-modules given by

$$
0 \rightarrow I \rightarrow \mathrm{g}_{0} \rightarrow K \rightarrow 0
$$


Tensoring both $\left(\Lambda_{f}\right)_{D}$ we get the exact sequence

$$
\left(\Lambda_{f}\right)_{D} \otimes_{g_{0}} I \stackrel{\eta}{\rightarrow} \Lambda_{f} \rightarrow\left(\Lambda_{f}\right)_{D} \otimes_{\mathbb{g}_{0}} K \rightarrow 0 .
$$

The image of $\eta$ is $\Lambda_{f} D(I)$ which by Lemma 5.1 is the same as $J$. Thus $\left(\Lambda_{f}\right)_{D} \otimes_{\mathfrak{B}_{0}} K \approx \Lambda_{f} / J \approx \mathfrak{g}_{f}$.

Consider now the natural isomorphisms

$$
\begin{aligned}
A \otimes_{\Lambda_{f}}\left(\Lambda_{f}\right)_{D} \otimes_{B_{0}} X & \approx A_{D} \otimes_{B_{0}} X, \\
\operatorname{Hom}_{\Lambda_{f}}\left(\left(\Lambda_{f}\right)_{D} \otimes_{B_{0}} X, A\right) & \approx \operatorname{Hom}_{B_{0}}\left(X,{ }_{D} A\right) .
\end{aligned}
$$

Passing to homology we get the rest of the assertions of the proposition.

From now on, we assume that $\mathfrak{g}$ has a finite $K$-base. Let $h: \mathfrak{g} \rightarrow K$ be defined as in the previous section by $h(x)=\operatorname{Tr}(\operatorname{Ad} x)$. It is clear, using Corollary 3.3, that there exists a $K$-algebra automorphism $\alpha_{f}$ of $g_{f}$ which satisfies for every $x \in \mathfrak{g}$

$$
\alpha_{f}\left(i_{f}(x)\right)=i_{f}(x)+h(x) .
$$

Let $\bar{\alpha}_{f}=\alpha_{f} \otimes i: \Lambda_{f} \rightarrow \Lambda_{f}, i$ denoting the identity map of $\mathfrak{g}_{f}$. It is clear that the following diagram is commutative.

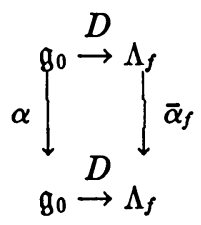

THEOREM 5.4. If $\mathfrak{g}$ is $K$-free with a base consisting of $n$-elements, then

$$
w \operatorname{dim}_{K} \mathfrak{g}_{f}=\operatorname{dim}_{K} \mathfrak{g}_{f}=n .
$$

Proof. Combining Proposition 5.3 and Theorem 4.1, we see that for any two sided $\mathfrak{g}_{f}$-module $A$, we have

$$
H_{r}\left(\mathfrak{g}_{f}, A\right)=H^{r}\left(\mathfrak{g}_{f}, A\right)=(0) \quad \text { for } r>n .
$$

On the other hand, by Proposition 5.3,

$$
\begin{aligned}
H_{n}\left(\mathfrak{g}_{f}, A_{\bar{\alpha}_{f}}\right) & \approx H_{n}\left(\mathfrak{g}, A_{\bar{\alpha}_{\rho} \circ D}\right) \\
& =H_{n}\left(\mathfrak{g}, A_{D_{\circ} \circ \alpha}\right) .
\end{aligned}
$$

Since $A_{D \circ \alpha}=\left(A_{D}\right)_{\alpha}$, it follows from Theorem 4.1 that $H_{n}\left(\mathfrak{g}, A_{D \circ \alpha}\right)$ is the annihilator of $I$ in $A_{D}$. This is the set of all elements of $A$ annihilated by $D(x)=i_{f}(x) \otimes 1-1 \otimes i_{f}(x)^{*}$; i.e. the set of all $a \in A$ with $\lambda a=a \lambda$ for $\lambda \in \mathfrak{g}_{f}$. Taking $A=\mathfrak{g}_{f}$, we find $H_{n}\left(\mathfrak{g}_{f},\left(\mathfrak{g}_{f}\right)_{\bar{\alpha}_{f}}\right)=$ centre of $\mathfrak{g}_{f} \neq(0)$.

6. The abelian case. The main objective of this section is to prove the following 
TheOREM 6.1. Let $\mathfrak{g}$ be a nonzero abelian Lie algebra over a field $K$ and let $f \in Z^{2}(\mathfrak{g}, K)$. Then the following conditions are equivalent.

(i) $H^{i}\left(g_{f}, g_{f}\right)=(0)$ for all $i>0$.

(ii) $H^{1}\left(\mathfrak{g}_{f}, \mathfrak{g}_{f}\right)=(0)$.

(iii) For each $\lambda \in \operatorname{Hom}_{K}\left(\mathfrak{g}, \mathfrak{g}_{f}\right)$ such that

$$
[[x, \lambda(y)]]=[[y, \lambda(x)]] \quad \text { for all } x, y \in \mathfrak{g},
$$

there exists an element $\lambda_{0} \in \mathfrak{g}_{f}$ such that

$$
\left[\left[x, \lambda_{0}\right]\right]=\lambda(x) \quad \text { for all } x \in \mathfrak{g} .
$$

(iv) $K$ is of characteristic zero, $\mathfrak{g}$ is of finite even dimension over $K$ and there exists a $K$-base of $\mathfrak{g}$ denoted by $y_{1}, z_{1}, \cdots, y_{n}, z_{n}$ such that

$$
f\left(y_{i}, y_{j}\right)=f\left(z_{i}, z_{j}\right)=0 ; \quad f\left(y_{i}, z_{j}\right)=\delta_{i j}, \quad i, j=1, \cdots, n .
$$

Proof. In stating condition (iii) we identified each element $x \in g$ with the element $i_{f} x \in g_{f}$ and thus regarded $g$ as a subspace of $g_{f}$. The notation $[[\alpha, \beta]]$ is used as usual for the commutator $\alpha \beta-\beta \alpha$. From the general rule

$$
\left[\left[\alpha, \beta_{1} \cdots \beta_{n}\right]\right]=\sum_{1 \leq i \leq n} \beta_{1} \cdots\left[\left[\alpha, \beta_{i}\right]\right] \cdots \beta_{n},
$$

we deduce for $x, y_{1} \cdots y_{n} \in g$

$$
\left[\left[x, y_{1} \cdots y_{n}\right]\right]=\sum_{1 \leq i \leq n} f\left(x, y_{i}\right) y_{1} \cdots y_{i} \cdots y_{n},
$$

where $\hat{y}_{i}$ indicates that $y_{i}$ is omitted. In particular, for $x, y \in \mathfrak{g}, n \geqq 0$,

$$
\left[\left[x, y^{n}\right]\right]=n f(x, y) y^{n-1} \text {. }
$$

Clearly, (i) $\Rightarrow$ (ii).

(ii) $\Leftrightarrow$ (iii). By Proposition 5.3,

$$
H^{1}\left(\mathfrak{g}_{f}, \mathfrak{g}_{f}\right) \approx H^{1}\left(\mathfrak{g}, D\left(\mathfrak{g}_{f}\right)\right) .
$$

Since for $x \in \mathfrak{g}, t \in g_{f}$ we have $D(x) t=[[x, t]]$, condition (6.1) states that $\lambda: \mathfrak{g} \rightarrow \mathfrak{g}_{f}$ is a derivation of $\mathfrak{g}$ in ${ }_{D}\left(g_{f}\right)$, while (6.2) expresses that $\lambda$ is an inner derivation. Thus (iii) is equivalent to $H^{1}\left(\mathfrak{g}_{f}, \mathfrak{g}_{f}\right)=(0)$.

(iii) $\Rightarrow$ (iv). Let $\phi \in \operatorname{Hom}_{K}(\mathfrak{g}, K)$. With the natural imbedding of $K$ in $\mathfrak{g}_{f}$, we regard $\phi$ as a $K$-homomorphism $\mathfrak{g} \rightarrow \mathfrak{g}_{f}$. Moreover, condition (6.1) is trivially satisfied. Thus, there exists $\lambda_{0} \in g_{f}$ with $\left[\left[x, \lambda_{0}\right]\right]=\phi(x)$ for all $x \in g$. We shall show that $\lambda_{0}$ may be chosen in $g$. To this end, choose a $K$-base $\left\{x_{\alpha}\right\}$ of $g$ indexed by a totally ordered set $I$. Then, by Theorem $2.6, \lambda_{0}$ can be written uniquely as

$$
\lambda_{0}=k+\sum_{\alpha} k_{\alpha} x_{\alpha}+\sum_{J} k_{J} x_{J}
$$


where $J$ are increasing and of length at least two. Let $y=\sum k_{\alpha} x_{\alpha}$ and $\gamma=\sum k_{J} x_{J}$. Then

$$
\phi(x)=\left[\left[x, \lambda_{0}\right]\right]=[[x, y]]+[[x, \gamma]] .
$$

Since $\phi(x) \in K$ and $[[x, y]]=f(x, y) \in K$ it follows that $[[x, \gamma]] \in K$. However, by $(6.4)[[x, \gamma]]$ is a $K$-linear combination of $x_{J^{\prime}}$, where $J^{\prime}$ are increasing of length at least one. Thus $[[x, \gamma]]=0$. Consequently,

$$
\phi(x)=[[x, y]]=f(x, y) \quad \text { for all } x \in \mathfrak{g} .
$$

This implies that the $K$-linear map

$$
\phi_{f}: \mathfrak{g} \rightarrow \operatorname{Hom}_{K}(\mathfrak{g}, K)
$$

given by $\phi_{f}(x)(y)=f(y, x)$ is an epimorphism. This rules out the possibility of $\mathfrak{g}$ having an infinite base. Since $\mathfrak{g}$ and $\operatorname{Hom}_{K}(\mathfrak{g}, K)$ have equal dimension, it is clear then that $\phi_{f}$ is an isomorphism and that $f$ is nondegenerate. It then follows from a well-known result on nondegenerate skew-symmetric forms $\left[4\right.$, p. 395] that $g$ has even dimension over $K$ and that it has a base $y_{1}, z_{1}, \cdots$, $y_{n}, z_{n}$, such that (6.3) is valid.

Suppose now that $K$ is of characteristic $p \neq 0$. Define $\lambda \in \operatorname{Hom}_{K}\left(\mathfrak{g}, \mathfrak{g}_{f}\right)$ setting $\lambda\left(y_{1}\right)=z_{1}^{p-1}$ and setting $\lambda$ as zero on the remaining basis elements $z_{1}, y_{2}, \cdots, y_{n}, z_{n}$. Then $[[x, \lambda(y)]]=0$ for all $x, y \in g$, so that (6.1) holds. There exists then $\lambda_{0} \in g_{f}$ with $\left[\left[x, \lambda_{0}\right]\right]=\lambda(x)$ for all $x \in g$. In particular, $\left[\left[y_{1}, \lambda_{0}\right]\right]=z_{1}^{p-1}$. By Theorem 2.6, we may write $\lambda_{0}$ uniquely as

$$
\lambda_{0}=\sum_{i, j \geq 0} \stackrel{i}{y_{1}^{j} z_{1} t_{i j}}
$$

where each $t_{i j}$ is a linear combination of increasing monomials in $y_{2}, z_{2}, \cdots$, $y_{n}, z_{n}$. Then, by (6.4) and (6.5),

$$
z_{1}^{p-1}=\left[\left[y_{1}, \lambda_{0}\right]\right]=\sum j y_{1}^{i} z_{1}^{j-1} t_{i j}
$$

because $\left[\left[y, t_{i j}\right]\right]=0$. Since $z_{1}^{p-1}$ occurs on the right-hand side with coefficient zero, we obtain a contradiction.

(iv) $\Rightarrow(\mathrm{i})$. We first consider the case $n=1$. Then $g$ is an abelian Lie algebra with a base $y, z$ such that $f(y, z)=1$ and $g_{f}$ is the algebra with two generators $y, z$ with the relation $[[y, z]]=1$.

To prove that $H^{1}\left(\mathfrak{g}_{f}, \mathfrak{g}_{f}\right)=(0)$, it suffices to show that (iii) holds. Let then $\lambda \in \operatorname{Hom}_{K}\left(g, g_{f}\right)$ with

$$
\lambda(y)=\sum_{i, j \geq 0} a_{i j} y^{i} z^{j}, \quad \lambda(z)=\sum_{i, j \geq 0} b_{i j} y^{i} z^{j} ; \quad a_{i j}, b_{i j} \in K .
$$

By (6.5),

$$
[[y, \lambda(z)]]=\sum j b_{i j} y^{i} z^{j-1}, \quad[[z, \lambda(y)]]=-\sum i a_{i j} y^{i-1} z^{2} .
$$


Thus, condition (6.1) becomes

$$
-(i+1) a_{i+1, j}=(j+1) b_{i j+1}, \quad i, j \geqq 0 .
$$

Define

$$
\lambda_{0}=\sum\left(a_{0 j} /(j+1)\right) z^{j+1}-\sum\left(b_{i j} /(i+1)\right) y^{i+1} z^{j} .
$$

Then, direct computation yields $\left[\left[y, \lambda_{0}\right]\right]=\lambda(y)$ and $\left[\left[z, \lambda_{0}\right]\right]=\lambda(z)$. Thus, (iii) holds and $H^{1}\left(\mathfrak{g}_{f}, \mathfrak{g}_{f}\right)=(0)$.

By Proposition 5.3 and Theorem 4.1, we have $H^{2}\left(\mathfrak{g}_{f}, \mathfrak{g}_{f}\right) \approx \mathfrak{g}_{f} / C$ where $C$ is the $K$-submodule of $g_{f}$ generated by $[[x, t]], x \in g, t \in g_{j}$. Since $y^{i} z_{j}$ $=\left[\left[y, y^{i} z^{j+1} /(j+1)\right]\right]$, it follows that $C=g_{f}$ and thus $H^{2}\left(g_{f}, g_{f}\right)=(0)$. Since $H^{i}\left(\mathfrak{g}_{f}, \mathfrak{g}_{f}\right)=(0)$ for $i>2$ by Theorem 5.4 , it follows that $H^{i}\left(\mathfrak{g}_{f}, \mathfrak{g}_{f}\right)=(0)$ for $i>0$.

Having disposed of the case $n=1$, let $n>1$. Let $\Lambda$ be the subalgebra of $g_{f}$ generated by $y_{1}, z_{1}$ and $\Gamma$ the subalgebra generated by $y_{2}, z_{2}, \cdots, y_{n}, z_{n}$. Then $g_{f}$ may be identified with the tensor product $\Lambda \otimes_{K} \Gamma$. Since $\Lambda$ and $\Gamma$ are $K$-projective, the $V$-product of $[1$, p. 216] applies here to yield a homomorphism

$$
\mathrm{V}: \sum_{p+q=m} H^{p}(\Lambda, \Lambda) \otimes H^{q}(\Gamma, \Gamma) \rightarrow H^{m}\left(\mathfrak{g}_{f}, \mathfrak{g}_{f}\right) .
$$

Since $\Lambda$ and $\Gamma$ are both Noetherian (Corollary 2.9), $V$ is an isomorphism by $\left[1\right.$, p. 209, Theorem 3.1]. Since we have already shown that $H^{p}(\Lambda, \Lambda)=(0)$ for $p>0$, we obtain

$$
H^{m}\left(\mathfrak{g}_{f}, \mathfrak{g}_{f}\right) \approx H^{0}(\Lambda, \Lambda) \otimes H^{m}(\Gamma, \Gamma) .
$$

The desired conclusion now follows by induction on $n$.

REMARK 6.2. The above theorem implies in particular that for an abelian Lie algebra $g$ over a field $K$ and $f \in Z^{2}(\mathfrak{g}, K), H^{1}\left(\mathfrak{g}_{f}, \mathfrak{g}_{f}\right)=(0)$ if and only if $K$ is of characteristic zero and $g_{f} \approx \otimes_{n} \Lambda$, where $\Lambda$ is the $K$-algebra on two generators $y, z$ with the relation $[[y, z]]=1$. If $K$ is the field of complex numbers, $\Lambda$ plays a fundamental role in quantum mechanics [3].

\section{APPENDiX: Filtered Algebras AND MOdUles}

We include here the proofs of some results on filtered modules and algebras, which have been utilized in the preceding sections.

Let $K$ be a commutative ring with unit element. An increasingly filtered $K$-module $A$ is a $K$-module $A$ and a sequence $\left\{F_{n} A\right\}_{n \in Z}$ of $K$-submodules $F_{n} A$ of $A$ such that for all $n, F_{n} A \subseteq F_{n+1} A$ and $\bigcup_{n \in Z} F_{n} A=A$. For the purpose of this appendix we shall also assume that $F_{n} A=(0)$ for $n<0$ and we shall refer to $A$ briefly as a filtered $K$-module. We set for convenience $F_{\infty} A=A$. Let $E^{0}(A)$ be the graded $K$-module whose $n$th component $E_{n}^{0}(A)$ is $F_{n} A / F_{n-1} A$. We call $E^{0}(A)$ the graded $K$-module associated with the filtered $K$-module $A$.

Let $A$ and $B$ be two filtered $K$-modules. A map $f: A \rightarrow B$ is called a homomorphism of filtered modules if it is a $K$-linear map such that $f\left(F_{p} A\right) \subseteq F_{p} B$ 
for all $p \in Z$. It is clear that such a map induces a mapping $E^{0}(f): E^{0}(A)$ $\rightarrow E^{0}(B)$ of graded modules.

A filtered $K$-algebra $\Lambda$ is a $K$-algebra in the usual sense which is also a filtered $K$-module such that for all $p, q$ we have $F_{p} \Lambda \cdot F_{q} \Lambda \subseteq F_{p+q} \Lambda$. It is clear that if $\Lambda$ is a filtered $K$-algebra, $E^{0}(\Lambda)$ acquires the structure of a graded $K$ algebra.

Let $\Lambda$ be a filtered $K$-algebra. A filtered right (or left) $\Lambda$-module is a right (or left) $\Lambda$-module in the usual sense which is also a filtered $K$-module such that for all $p, q$ we have $F_{p} A \cdot F_{q} \Lambda \subseteq F_{p+q} A$ (or $F_{q} \Lambda \cdot F_{p} A \subseteq F_{p+q} A$ ). If $A$ is a filtered $\Lambda$-module, it is clear that $E^{0}(A)$ is a graded $E^{0}(\Lambda)$-module.

Proposition 1. Let $A$ and $B$ be filtered $K$-modules and $\theta: A \rightarrow B$ a homomorphism of filtered modules. If $E^{0}(\theta): E^{0}(A) \rightarrow E^{0}(B)$ is an isomorphism, then $\theta$ is an isomorphism.

Proof. Let $\theta_{p}: F_{p} A \rightarrow F_{p} B$ denote the $K$-linear map induced by $\theta$. Clearly $\theta_{0}$ is an isomorphism. We assume therefore by induction that $\theta_{p-1}$ is an isomorphism. From the commutativity of the diagram

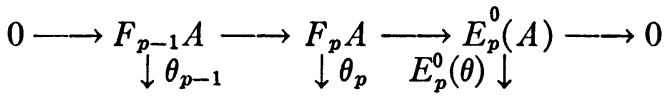

$$
\begin{aligned}
& 0 \longrightarrow F_{p-1} B \longrightarrow F_{p} B \longrightarrow E_{p}^{0}(B) \longrightarrow 0
\end{aligned}
$$

and the fact that $\theta_{p-1}$ and $E_{p}^{0}(\theta)$ are isomorphisms, it is easily seen that $\theta_{p}$ is also an isomorphism. Since $p$ is arbitrary, the assertion of the proposition is clear.

Proposition 2. Let $A$ be a filtered right module over a filtered $K$-algebra $\Lambda$. If $E^{0}(A)$ is $E^{0}(\Lambda)$-free in the graded sense, then $A$ is $\Lambda$-free. In fact, if $\left(b_{p, \alpha}\right)$, $b_{p, \alpha} \in E_{p}^{0}(A), p \geqq 0$ denotes a homogeneous base of $E^{0}(A)$ and if $a_{p, \alpha}$ are arbitrary liftings of $b_{p, \alpha}$ to $F_{p} A$, then $\left(a_{p, \alpha}\right)$ form $a \Lambda$-base for $A$.

Proof. For any filtered $\Lambda$-module $B$ and any nonzero $b \in B$, we set $\nu(b)$ $=$ the least integer such that $b \in F_{\nu(b)} B$. We denote by $b$ the element represented by $b$ in $F_{\nu(b)} B / F_{\nu(b)-1} B$. Clearly $b \neq 0$ implies $b \neq 0$.

We first prove that the $\left(a_{p, \alpha}\right)$ generate $A$ as a right $\Lambda$-module. Let $a \in A$. If $a=0$, it is trivially a linear combination of the $a_{p, \alpha}$. Let then $a \neq 0$ and set $\nu(a)=p$. Clearly we have,

$$
\bar{a}=\sum_{j, \alpha} b_{j, \alpha} t_{j, \alpha}, \quad t_{j, \alpha} \in E^{0}(\Lambda)
$$

Let $\lambda_{j, \alpha} \in \Lambda$ be such that $t_{j, \alpha}=\bar{\lambda}_{j, \alpha}$. We then have

$$
a=\sum a_{j, \alpha} \lambda_{j, \alpha} \bmod F_{p-1} A .
$$

It is now evident, using induction on $p$, that the $\left(a_{p, \alpha}\right)$ generate $A$. We prove now that they are $\Lambda$-linearly independent. Suppose in fact 


$$
\sum a_{j, \alpha} \lambda_{j, \alpha}=0,
$$

such that all the $\lambda_{j, \alpha}$ occurring in the sum on the left-hand side are nonzero. We then get

$$
\sum b_{j, \alpha} \bar{\lambda}_{j, \alpha}=0 .
$$

Since the $\left(b_{p, \alpha}\right)$ are $E^{0}(\Lambda)$-linearly independent, we get $\bar{\lambda}_{j, \alpha}=0$, a contradiction.

In what follows, for any filtered module $A$, we set $A_{p}=F_{p} A$ for convenience.

Lemma 3. If a filtered $K$-module $A$ is such that $E^{0}(A)$ is $K$-flat, then for any $p, q$ (including $p=\infty) A_{p} / A_{q}$ is $K$-flat.

Proof. Let us assume by induction that $A_{r} / A_{q}$ is $K$-flat for $r<p$. Consider the exact sequence

$$
0 \rightarrow A_{p-1} / A_{q} \rightarrow A_{p} / A_{q} \rightarrow E_{p}^{0}(A) \rightarrow 0 .
$$

By assumption $E_{p}^{0}(A)$ is $K$-flat and by induction $A_{p-1} / A_{q}$ is $K$-flat. It is now clear that $A_{p} / A_{q}$ is $K$-flat. Since $A / A_{q}$ is the direct-limit of $A_{p} / A_{q}$, the case $p=\infty$ follows.

From now on, $A$ will always denote a filtered $K$-module such that $E^{0}(A)$ is $K$-flat. Let $B$ be an arbitrary filtered $K$-module. We set $C_{p, q}=A_{p} \otimes_{K} B_{q}$. Since, by Lemma 3, $A / A_{p}$ is $K$-flat, the map $C_{p, q} \rightarrow C_{\infty, q}$ defined in the obvious way is a monomorphism. Since $A$ is $K$-flat, the map $C_{\infty, q} \rightarrow C_{\infty, \infty}$ is also a monomorphism. We thus have a $K$-monomorphism $C_{p, q} \rightarrow C_{\infty, \infty}=A \otimes_{K} B$ and in what follows we shall regard this as an identification of $C_{p, q}$ with a $K$-submodule of $C_{\infty, \infty}$.

Lemma 4. $C_{p^{\prime}, q^{\prime}} \cap C_{p^{\prime \prime}, q^{\prime \prime}}=C_{\min \left(p^{\prime}, p^{\prime \prime}\right), \min \left(q^{\prime}, q^{\prime \prime}\right) \text {. }}$

Proof. We may assume $p^{\prime}<p^{\prime \prime}$ and $q^{\prime}>q^{\prime \prime}$ for this is the only nontrivial case to be considered. Consider the following commutative diagram of exact sequences.

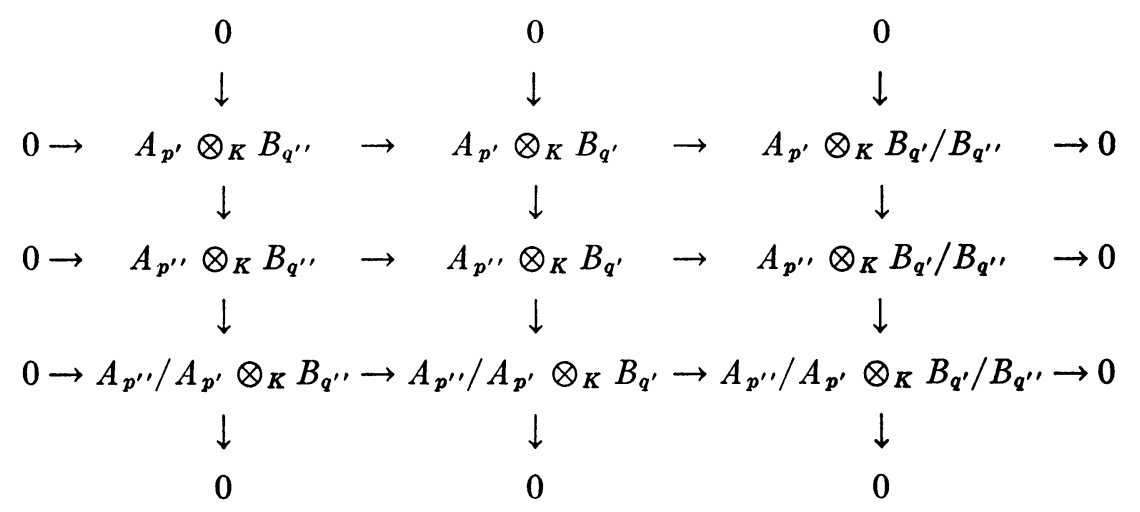


Chasing the above diagram in the obvious way we get

$$
\left(A_{p^{\prime}} \otimes_{K} B_{q^{\prime}}\right) \cap\left(A_{p^{\prime \prime}} \otimes_{K} B_{q^{\prime \prime}}\right)=A_{p^{\prime}} \otimes_{K} B_{q^{\prime \prime}},
$$

which is the required assertion.

Lemma 5. $C_{0, q} \cap\left(\sum_{i} C_{m_{i}, n_{i}}\right)=C_{0, \min \left(q, \max n_{i}\right)}$.

Proof. Let $\max n_{\mathrm{i}}=n$. It is clear that the right-hand side is contained in the left-hand side. On the other hand, the left-hand side is clearly contained in $C_{0, q} \cap C_{\infty, n}=C_{0, \min (q, n)}$ by Lemma 4 .

LEMMA 6.

$$
\left(\sum_{i=1}^{n} C_{p_{i}, q_{i}}\right) \cap\left(\sum_{i=1}^{m} C_{r_{i}, s_{i}}\right)=\sum C_{\min \left(p_{i}, r_{j}\right), \min \left(q_{i}, s_{1}\right)} .
$$

Proof. We assume without loss of generality that

$$
\begin{gathered}
0 \leqq p_{1}<\cdots<p_{n} ; \quad q_{1}>\cdots>q_{n} \geqq 0 . \\
0 \leqq r_{1}<\cdots<r_{m} ; \quad s_{1}>\cdots>s_{m} \geqq 0 . \\
q_{1} \geqq s_{1} .
\end{gathered}
$$

Since all the modules in question are submodules of $A \otimes_{K} B_{q_{1}}$, we may assume $B=B_{q_{1}}$. Let $f: A \rightarrow A / A_{0}$ be the factorization map and let $\bar{f}=f \otimes i: A \otimes_{K} B_{q_{1}}$ $\rightarrow A / A_{0} \otimes_{K} B_{q_{1}}$, where $i: B_{q_{1}} \rightarrow B_{q_{1}}$ is the identity map. Let $C=$ the left-hand side of $\left({ }^{*}\right)$ and $D=$ right-hand side. Obviously $D \subseteq C$. Clearly it is enough to prove

$$
\begin{aligned}
(\operatorname{Ker} \bar{f}) \cap C & =(\operatorname{Ker} \bar{f}) \cap D . \\
\bar{f}(C) & =\bar{f}(D) .
\end{aligned}
$$

Ker $\bar{f}=A_{0} \otimes_{K} B_{q_{1}}=C_{0, q_{1}}$. Using Lemma 5 , we see that

$$
C_{0, q_{1}} \cap C=C_{0, s_{1}}=C_{0, q_{1}} \cap D .
$$

Hence (1) is proved. We now prove (2). Since $\operatorname{Ker} \bar{f}=C_{0, q_{1}} \subset \sum C_{p_{i}, q_{i}}$, it is clear that

$$
\begin{aligned}
\bar{f}(C) & =\bar{f}\left(\sum C_{p_{i}, q_{i}}\right) \cap \bar{f}\left(\sum C_{r i, s_{i}}\right) \\
& =\left(\sum \bar{f}\left(C_{p_{i}, q_{i}}\right)\right) \cap\left(\sum \bar{f}\left(C_{r_{i}, \varepsilon_{i}}\right)\right)
\end{aligned}
$$

Also,

$$
\bar{f}(D)=\sum \bar{f}\left(C_{\min \left(p_{i}, r_{j}\right), \min \left(q_{i}, s_{j}\right)}\right) .
$$

By Lemma $3, \bar{A}=A / A_{0}$ is $K$-flat. We make $\bar{A}$ into a filtered $K$-module by setting $\bar{A}_{p}=A_{p+1} / A_{0}$. Let $\bar{C}_{p, q}=\bar{A}_{p} \otimes_{K} B_{q}$. Thus $\bar{f}\left(C_{p, q}\right)=\bar{C}_{p-1, q}$. Using the above, the condition (2) reads.

$$
\left(\sum \bar{C}_{p_{i}-1, q_{i}}\right) \cap\left(\sum \bar{C}_{r_{i}-1, s_{i}}\right)=\sum \bar{C}_{\min \left(p_{i}-1, r_{j}-1\right), \min \left(q_{i}, s_{j}\right)} .
$$


But this is precisely the assertion of the lemma for $\bar{A}$ with all the $p_{i}$ and $r_{i}$ decreased by 1 . An obvious induction now finishes the proof.

Let $A$ be a filtered $K$-module such that $E^{0}(A)$ is $K$-flat. Let $B$ be an arbitrary filtered $K$-module. If we set $\left(A \otimes_{K} B\right)_{r}=\sum_{p+q-r} C_{p, q}$, it is clear that $\left(A \otimes_{K} B\right)_{r}$ defines a $K$-filtration of $A \otimes_{K} B$.

We make the bigraded $K$-module $E^{0}(A) \otimes_{K} E^{0}(B)$ into a singly graded module by setting as usual

$$
\left(E^{0}(A) \otimes_{K} E^{0}(B)\right)_{r}=\sum_{p+q=r} E_{p}^{0}(A) \otimes_{K} E_{q}^{0}(B) .
$$

We have for each $p, q$ the exact sequences

$$
\begin{aligned}
& 0 \rightarrow A_{p-1} \rightarrow A_{p} \rightarrow E_{p}^{0}(A) \rightarrow 0, \\
& 0 \rightarrow B_{q-1} \rightarrow B_{q} \rightarrow E_{q}^{0}(B) \rightarrow 0,
\end{aligned}
$$

and hence an exact sequence

$$
C_{p, q-1}+C_{p-1, q} \rightarrow C_{p, q} \stackrel{\alpha_{p, q}}{\longrightarrow} E_{p}^{0}(A) \otimes_{K} E_{q}^{0}(B) \rightarrow 0 .
$$

Let

$$
i_{p, q}: E_{p}^{0}(A) \otimes_{K} E_{q}^{0}(B) \rightarrow E_{p+q}^{0}\left(A \otimes_{K} B\right)
$$

be the unique map which makes the diagram

$$
\begin{array}{ccc}
C_{p, q-1}+C_{p-1, q} & C_{p, q} & \stackrel{\alpha_{p, q}}{\longrightarrow} E_{p}^{0}(A) \otimes_{K} E_{q}^{0}(B) \rightarrow 0 \\
\downarrow & \downarrow & \downarrow i_{p, q} \\
\sum_{i+j=p+q-1} C_{i, j} \longrightarrow & \sum_{i+j=p+q} C_{i, j} \stackrel{\beta_{p+q}}{\longrightarrow} E_{p+q}^{0}\left(A \otimes_{K} B\right) \longrightarrow 0
\end{array}
$$

(where $\beta_{p+q}$ is the factorization map and the unnamed maps are the various inclusions), commutative. We thus have for each integer $r$, the map

$$
i_{r}:\left(E^{0}(A) \otimes_{K} E^{0}(B)\right)_{r} \rightarrow E_{r}^{0}\left(A \otimes_{K} B\right) .
$$

Proposition 7. Let $A$ be a filtered $K$-module such that $E^{0}(A)$ is $K$-flat. If we make $A \otimes_{K} B$ into a filtered $K$-module by setting

$$
\left(A \otimes_{K} B\right)_{r}=\sum_{p+q=r} A_{p} \otimes_{K} B_{q},
$$

then the mapping

$$
i: E^{0}(A) \otimes_{K} E^{0}(B) \rightarrow E^{0}\left(A \otimes_{K} B\right)
$$


is an isomorphism of graded $K$-modules. If $A$ and $B$ are filtered $K$-algebras, the above map is an isomorphism of graded $K$-algebras.

Proof. To prove the first assertion of the proposition, we need only show that the map $i_{r}$ is an isomorphism for each $r$. If $y=\sum_{p+q=r} \beta_{p+q}\left(t_{p, q}\right), t_{p, q} \in C_{p, q}$ denotes an arbitrary element of $E_{r}^{0}\left(A \otimes_{K} B\right)$, then the element $x=\sum \alpha_{p, q}\left(t_{p, q}\right)$ $\in\left(E^{0}(A) \otimes_{K} E^{0}(B)\right)_{r}$ is such that $i_{r}(x)=y$. Hence $i_{r}$ is an epimorphism. On the other hand, suppose that $x=\sum_{p+q=r} \alpha_{p, q}\left(t_{p, q}\right), t_{p, q} \in C_{p, q}$ is such that

$$
i_{r}(x)=\sum_{p+q=r} \beta_{p+q}\left(t_{p, q}\right)=\beta_{r}\left(\sum t_{p, q}\right)=0 \text {. }
$$

Then

$$
\sum t_{p, q} \in \operatorname{Ker} \beta_{r}=\sum_{i+j=r-1} C_{i, j}
$$

Thus, for any $p, q$ with $p+q=r$, we have

$$
t_{p, q} \in \sum_{i \neq p, i+j=r} C_{i, j}+\sum_{i+j=r-1} C_{i, j} .
$$

Thus, we have

$$
t_{p, q} \in C_{p, q} \cap\left(\sum_{i \neq p, i+j=r} C_{i, j}+\sum_{i+j=r-1} C_{i, j}\right)=C_{p, q-1}+C_{p-1, q},
$$

the equality being a consequence of Lemma 6 . Thus, $\alpha_{p, q}\left(t_{p, q}\right)=0$ and hence $x=0 . i_{r}$ is therefore a monomorphism. Hence $i_{r}$ is an isomorphism.

The second assertion of the proposition is clear.

\section{BiBLIOGRAPHY}

1. H. Cartan and S. Eilenberg, Homological algebra, Princeton University Press, 1956.

2. C. Chevalley and S. Eilenberg, Cohomology theory of Lie groups and Lie algebras, Trans. Amer. Math. Soc. vol. 63 (1948) pp. 85-124.

3. H. Weyl, The theory of groups and quantum mechanics, Dover Press.

Columbia University, NeW YORK, NEW YORK

Tata Institute of Fundamental Research, BOMBAY, INDIA 